\title{
Effect of Triage Nurse Initiated Radiography Using the Ottawa Ankle Rules on Emergency Department Length of Stay at a Tertiary Centre
}

\author{
Wailliam W. Lee, $\mathrm{MD}^{*}{ }^{\dagger}$; Lyne Filiatrault, $\mathrm{MDCM}^{*}{ }^{\dagger}$; Riyad B. Abu-Laban, MD, MHSc ${ }^{{ }^{\dagger *}}$; Anita Rashidi, \\ $\mathrm{BSc}^{\S}$; Lawrence Yau, BMSc*; Nancy Liu, BSc ${ }^{\S}$
}

\section{ABSTRACT}

Objective: To determine the effect of triage nurse initiated radiographs using the Ottawa Ankle Rules (OAR) on emergency department (ED) throughput. We hypothesized OAR use would reduce median ED length of stay (LOS) by 25 minutes or more. Methods: A randomized controlled trial was conducted at a tertiary centre ED with an annual census of over 90,000 patients. Adult patients presenting within 10 days of isolated blunt ankle trauma were eligible. Participants were randomly assigned to standard triage or OAR application by 15 explicitly trained triage nurses. Our primary outcome was ED LOS. Secondary outcomes included triage nurses' and patients' satisfaction. A power calculation indicated 142 patients were required. The Mann-Whitney $U$ test was used to compare the medians between the two groups.

Results: Of 176 patients with blunt ankle injury screened, 146 were enrolled (83.0\%); baseline characteristics in the two groups were similar. The median/mean ED LOS in the control and OAR groups were 128/143 minutes and 108/115 minutes respectively (median difference 20 minutes; $p=0.003$ ). Agreement in OAR use between emergency physicians and nurses was moderate (kappa $0.46 / 0.77$ for foot/ankle rule components), and satisfaction of both nurses and participants was high.

Conclusion: Triage nurse initiated radiography using OAR leads to a statistically significant decrease of 20 minutes in the median ED LOS at a tertiary care centre. The overall impact of implementing such a process is likely site-specific, and the decision to do so should involve consideration of the local context.

\section{RÉSUMÉ}

Objectif: L'étude visait à déterminer l'effet du triage des patients par le personnel infirmier par la prescription de radiographies selon les règles d'Ottawa concernant les blessures de la cheville, sur le flux des patients au service des urgences (SU). Selon I'hypothèse émise, I'application des règles permettrait de réduire la durée médiane de séjour (DS) de 25 minutes ou plus.
Méthode: Un essai comparatif à répartition aléatoire a été mené dans un SU d'un centre de soins tertiaires, qui reçoit plus de 90000 patients par année. Les adultes qui consultaient pour une tuméfaction isolée de la cheville dans les 10 jours suivant un trauma pouvaient être sélectionnés. Les participants étaient dirigés au hasard vers le groupe de triage habituel ou vers le groupe des règles d'Ottawa, appliquées par 15 infirmières ou infirmiers spécialement formés à cet effet. Le principal critère d'évaluation était la DS au SU. Les critères secondaires comprenaient le degré de satisfaction des patients et du personnel infirmier affecté au triage. D'après un calcul de la puissance de l'étude, le nombre nécessaire de patients à traiter devait atteindre 142. La comparaison des valeurs médianes entre les deux groupes a été effectuée à l'aide du test de Mann-Whitney.

Résultats: Sur 176 patients sélectionnés pour une contusion à la cheville, 146 ont participé à l'étude (83,0\%), et les caractéristiques de base dans les deux groupes étaient comparables. Les durées médiane et moyenne de séjour au SU dans le groupe témoin et dans le groupe expérimental se sont établies respectivement à 128 et 143 minutes et à 108 et 115 minutes (écart médian: 20 minutes; $p=0,003)$. Le degré de concordance dans I'application des règles entre les médecins d'urgence et le personnel infirmier était moyen (kappa: 0,46/0,77 pour les éléments des règles concernant le pied et la cheville) et le degré de satisfaction tant du personnel infirmier que des participants était élevé.

Conclusion: Le triage des patients par le personnel infirmier par la prescription de radiographies selon les règles d'Ottawa a permis de réduire d'une manière significative, soit de 20 minutes, la durée médiane de séjour dans un SU d'un centre de soins tertiaires. L'incidence générale de I'application des règles d'Ottawa est sans doute propre à chaque centre, et le contexte local devrait être pris en considération dans la décision relative à leur mise en œuvre.

Keywords: Ottawa Ankle Rules, Triage Nurses, Length of Stay, Ankle Radiographs, Radiology, Emergency Department

From the *Department of Emergency Medicine, Vancouver General Hospital, Vancouver Coastal Health, Vancouver, BC; †Department of Emergency Medicine, University of British Columbia, Vancouver, BC; $¥$ Center for Clinical Epidemiology and Evaluation, Vancouver Coastal Health Research Institute, Vancouver, BC; and §Faculty of Medicine, University of British Columbia, Vancouver, BC.

Correspondence to: Wailliam Lee, Vancouver General Hospital, Department of Emergency Medicine, 855 12 ${ }^{\text {th }}$ Ave W, Vancouver, BC, V5Z $1 \mathrm{M9}$; Email: wailliamlee37@gmail.com 


\section{INTRODUCTION}

Emergency department (ED) overcrowding remains an ongoing issue throughout North America. Numerous strategies have been implemented and explored in hopes of improving ED patient throughput. The Ottawa Ankle Rules (OAR) are a widely known and well-validated clinical decision rule for ankle radiography that has been shown to reduce cost and wait time without patient dissatisfaction or missed fractures. ${ }^{1-5}$ As a result, the OAR have gained widespread acceptance throughout the world. ${ }^{6-10}$

Numerous studies have examined nurses' interpretation and application of the OAR. ${ }^{11-14}$ It is clear that the use of the OAR by nurses results in similar diagnostic performance for fracture detection as when the rules are applied by physicians, ${ }^{15,16}$ and thus it is believed that emergency nurses can accurately determine which patients require radiographs in the setting of isolated blunt ankle injury. ${ }^{11,13,16}$ What remains unclear, however, is whether or not triage nurse initiated ankle radiography shortens ED patients' length of stay (LOS), a factor known to be correlated with patient satisfaction. ${ }^{17,18}$ To date, two studies have examined use of the OAR by triage nurses with ED LOS as the primary outcome. ${ }^{19,20}$ One was a retrospective case-control study conducted at an Accident \& Emergency Department in a small city. ${ }^{19}$ The other, although prospective and randomized, was carried out in an urgent care centre. ${ }^{20}$

Our objective was to determine the effect of triage nurse initiated radiographs using the OAR on ED LOS at a tertiary centre. For secondary (hypothesis generating) purposes, we also examined the number of fractures missed, agreement between triage nurses and emergency physicians (EPs) in the application of the OAR, and patients' and triage nurses' satisfaction with OAR application at triage. We hypothesized that triage nurse initiated radiograph in accordance with the OAR would reduce median ED LOS by 25 minutes or more.

\section{METHODS}

We conducted a prospective randomized controlled trial (RCT) from August 2012 to August 2013 in the ED of Vancouver General Hospital (VGH), an adult tertiary teaching and level 1 trauma centre with an annual ED census of over 90,000 patients. The VGH ED has a $23 \%$ admission rate and is staffed $24 / 7$ by specialist emergency physicians certified by the Royal College of Physicians and Surgeons of Canada. Patients aged 19 years or older presenting to our adult ED with an isolated blunt ankle trauma were potentially eligible. In an approach similar to the exclusion criteria of the original OAR study, patients were excluded if they had any of the following: an isolated skin injury; polytrauma; obvious fracture or deformity; neurovascular deficit; altered mental status; intoxication or were uncooperative; an $\mathrm{x}$-ray performed prior to their ED visit; pregnancy; a prior ED visit for the same injury; or injury occurred more than 10 days previously.

A prospective randomized control methodology was chosen over other designs, such as a before/after time series using historical controls, or single arm trial with concurrent control from another institution, due to the dynamic nature of the ED patient volume and staffing, and the numerous interventions at the study location that continue to be implemented to address patient wait time, all of which have the potential to significantly affect our primary outcome. Figure 1 provides a schematic of the study patients' management. A strategy similar to that of Fan and Woolfrey ${ }^{20}$ was employed. Through a one-on-one interactive computer training session, 15 participating ED triage nurses were explicitly trained by a single student research volunteer on the use of the OAR and the study enrollment criteria. Eligible patients were randomly assigned to either the OAR group or a control group with a 1:1 allocation ratio using a computerized random number generator. Randomization was performed using sealed envelopes stored at triage. Participants randomized to the OAR group were managed by triage nurses in accordance with the OAR and radiographs were ordered if indicated. Triage nurses recorded their assessment on a data collection form that included a visual

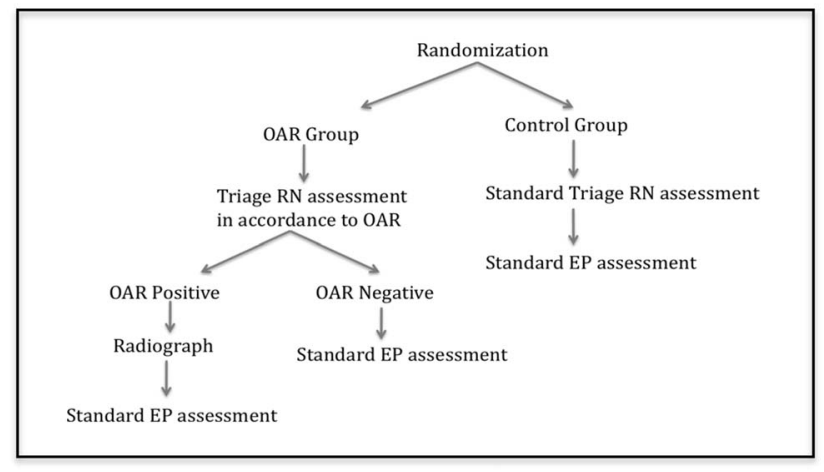

Figure 1. Schematic of study patients management. 
representation of the OAR. Participants randomized to the control group received standard assessment by triage nurses without the application of the OAR or nurse initiated radiographs. Although patients in both study groups were assessed by the same group of 15 triage nurses, contamination knowledge of the OAR in control group patients was precluded by the fact that standard triage nurse assessment does not include an in-depth ankle assessment, or the potential to order diagnostic imaging. In both scenarios, following triage, patients were evaluated as per standard practice by an EP (or a supervised staff member) on duty in the ambulatory section of the ED. Trained research students contacted patients by telephone at least 14 days after their ED visit to inquire about missed fractures and complete a patient satisfaction survey.

Data such as time variables and interventions were obtained from the hospital's PCIS (Patient Care Information System) electronic health record. The PCIS was also used to confirm radiograph order entry and to review the final radiology report for the presence of clinically significant ankle/foot fractures as defined by the original OAR study protocol. For patients who could not be contacted for follow-up, additional visits were reviewed in PCIS to assess for return visits for any missed fractures at one of three hospitals within the same health authority. Data was inputted into secured, password protected, computer spreadsheets.

The primary outcome was total median LOS in the $\mathrm{ED}$, defined as the difference between time of arrival in the ED (prior to actual triage) and the time of the EP's disposition. The latter was defined as one of the following: ED discharge, the time the EP authorized discharge, time of orthopedic consultation request, or time of CT order. The disposition of patients who were not immediately discharged was anchored by the time of orthopedic consultation or CT order, because both the orthopedic consultation process and diagnostic imaging introduced additional wait times that we considered to be separate from the ED flow. Secondary outcomes were the following: agreement between triage nurses and EPs in application of the OAR, missed fractures identified on patient telephone follow-up, triage nurse satisfaction in application of the OAR, and patient's satisfaction.

Pilot data indicated that the mean \pm SD LOS of patients presenting to the VGH ED with a chief complain of ankle injury was $121.3 \pm 59$ minutes. Using this information, we calculated that 71 patients were needed in each arm to have an $80 \%$ power to detect an improvement of 25 minutes in ED LOS. Our sample size calculation was two-tailed based on a $p$-value for significance of $<0.05$. We deemed a 25 -minute improvement to be clinically significant a priori, similar to the Fan and Woolfrey study. ${ }^{20}$

ED LOS was evaluated using descriptive statistics, including mean, median, standard deviation, and interquartile ranges. The difference between the medians of the OAR group and the control group were analyzed using the Mann-Whitney $U$ test, with $p$-values of less than 0.05 being considered statistically significant. Where appropriate, $95 \%$ confidence intervals were also calculated. Agreement between triage nurses and EPs in applying the OAR was evaluated using raw percent agreement and kappa statistics. Median scores were generated for patient and nurse satisfaction scores.

Approval was obtained from both the University of British Columbia Research Ethics Board and the Vancouver Coastal Health Research Institute. Consent was deemed to not be required for study participation, ${ }^{20}$ as the additional intervention conducted by triage nurses in the OAR group did not increase risk to patients and fell within the scope of practice of British Columbia registered nurses. Informed consent was obtained for participation in the telephone follow-up. The study was registered at Clinicaltrials.gov (registration \#NCT01654393).

\section{RESULTS}

From August 2012 to August 2013, 176 patients with ankle injuries were seen by one of the 15 participating triage nurses, 30 of whom were excluded. Figure 2 lists the reasons for exclusions and final number of patients in both control and OAR arms. Three patients were excluded because of protocol violations: one was a staff member whose care was expedited, one patient assigned to the control group had an $\mathrm{x}$-ray ordered by the triage nurse, and one assigned to the OAR group with a positive assessment did not have an $\mathrm{x}$-ray ordered by the triage nurse. The resulting final study sample size was 146 participants ( $83 \%$ of those screened): 75 in the control group and 71 in the OAR group. Baseline characteristics of the study groups are provided in Table 1, and were similar, with the exception of a higher proportion of CTAS 3 categorizations and lower proportion of CTAS 4 categorizations in the OAR group. 


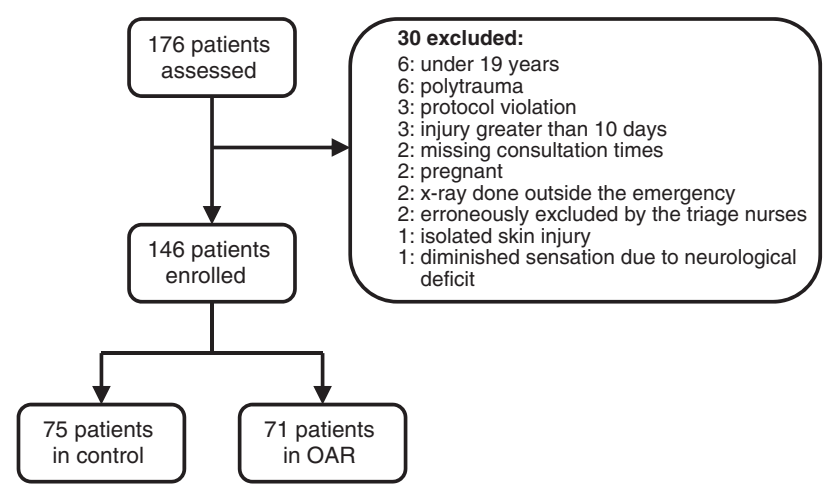

Figure 2. Case flow chart.

\begin{tabular}{lcc}
\hline \multicolumn{2}{l}{ Table 1. Baseline group characteristics } & \\
\hline Characteristics & Control & OAR \\
\hline Total patients per group & 75 & 71 \\
Age, $y r( \pm$ SD) & $35( \pm 13.7)$ & $38( \pm 15.7)$ \\
Gender, $n(\%)$ & \\
$\quad$ Male & $38(50.7)$ & $36(50.7)$ \\
Female & $37(49.3)$ & $35(49.3)$ \\
CTAS, $n(\%)$ & & \\
CTAS 3 & $16(21.3)$ & $26(36.6)$ \\
CTAS 4 & $59(78.7)$ & $45(63.4)$ \\
Overall x-rays ordered, $n(\%)$ & $67(89.3)$ & $69(97.2)$ \\
Fracture, $n(\%)$ & $20(26.7)$ & $21(29.6)$ \\
Ankle & $18(24.0)$ & $18(25.4)$ \\
Foot & $2(2.7)$ & $3(4.2)$ \\
Time of ED visit, $n(\%)$ & & \\
8:00 am to 11:59 am & $9(12.0)$ & $16(22.5)$ \\
12:00 pm to 5:59 pm & $29(38.7)$ & $17(23.9)$ \\
6:00 pm to 11:59 pm & $31(41.3)$ & $27(38.0)$ \\
12:00 am to 7:59 am & $6(8.0)$ & $11(15.5)$ \\
Time to telephone follow-up & & \\
Days $( \pm$ SD) & $81.3( \pm 70.9)$ & $81.1( \pm 54.3)$ \\
$n$ (\% of group completed) & $47(62.7)$ & $39(54.9)$ \\
\hline
\end{tabular}

Telephone follow-up was achieved on an average at 73 days (range 17-310 days). A total of 86 of 146 participants (59\%) completed the telephone survey: 47 (55\%) from the control group and 39 (45\%) from the OAR group. Telephone follow-up for missed fractures and patient satisfaction was not attempted for 17 participants living outside of British Columbia. Thirteen participants had wrong numbers and 27 subjects were deemed lost to telephone follow-up after at least five attempts. Three individuals declined to participate when reached by phone.

The median/mean ED LOS in the control and OAR groups was 128/143 minutes (range 31-364 minutes) and 108/115 minutes (range 37-293 minutes), respectively (median difference 20 minutes; $p=0.003$ ). Raw percentage agreement between EPs and triage nurses was $84 \%$ for the OAR ankle assessment and was $91 \%$ for the foot evaluation, with kappa values of 0.46 and 0.77 , respectively. Sixty-seven of the 75 patients $(89.3 \%)$ in the control group received $x$-rays ordered by the EP and 61 of the 71 patients (85.9\%) in the OAR group received $\mathrm{x}$-rays ordered by the triage nurse. Eight of the 10 patients in the OAR group, who did not have $\mathrm{x}$-rays ordered as they were deemed OAR negative by the triage nurse, had $\mathrm{x}$-rays ordered later by the EP. No ankle or foot fractures were missed by a failure of triage nurse to perform radiograph and captured by the EPs. There was one missed ankle fracture identified amongst the study population in a subject from the control group. Patient satisfaction survey results are outlined in Table 2. Participants from both groups were generally satisfied with the quality of care they had received, although those in the OAR group had a higher rating for their ED LOS. The median reduction in ED LOS that was deemed by participants in both group to be significant was 30 minutes. Overall, $88.4 \%$ of patients were comfortable having a triage nurse decide whether an $\mathrm{x}$-ray was needed and ordering it prior to the patient seeing the EP. Nurse satisfaction survey results are provided in Table 3. Although triage nurses were comfortable with applying the OAR and believed the intervention would reduce patients' ED LOS, 54\% were concerned that their resulting workload in triage would be increased.

\section{DISCUSSION}

In this prospective randomized control trial, we found that triage nurse initiated radiographs in accordance with the OAR shortened patient's median LOS by 20 minutes. This improvement likely arose from time saved by patients obtaining radiographs while waiting to be seen by an EP. In our institution, the time needed to obtain a radiograph is the same, whether it is initiated by an EP or a triage nurse. As EPs were blinded to the study patients' group assignment until they picked up the study patient's chart and opened the accompanying envelopes, they could not choose to assess the OAR patients ahead of the control group. Our intervention slightly underperformed when comparing our results to that of the Allerston and Justham study, which found an improvement of 25 minutes in median ED LOS, with 


\begin{tabular}{|c|c|c|}
\hline Characteristics & Control & OAR \\
\hline Completed telephone survey & 47 & 39 \\
\hline Length of stay ratings ${ }^{1}$ (median) & 6.0 & 7.5 \\
\hline Quality of care ratings ${ }^{1}$ (median) & 8.0 & 8.0 \\
\hline Length of stay ratings ${ }^{1}$ (mean) & 6.3 & 7.4 \\
\hline Quality of care ratings ${ }^{1}$ (mean) & 7.6 & 8.1 \\
\hline LOS $^{2}$ reductions preferred (in mins) & 30 & 30 \\
\hline
\end{tabular}

\begin{tabular}{|c|c|c|}
\hline Characteristics & \multicolumn{2}{|c|}{ Response } \\
\hline Comfort with applying $O A R^{2}$ & \multicolumn{2}{|c|}{8.5} \\
\hline \multirow[t]{2}{*}{ Likelihood of applying intervention post study ${ }^{3}$} & \multicolumn{2}{|c|}{8.7} \\
\hline & Yes & $\begin{array}{l}\text { No/ } \\
\text { Unsure }\end{array}$ \\
\hline Intervention increases triage nurse workload & 8 & 5 \\
\hline Believes intervention will reduce ED LOS & 11 & 2 \\
\hline Was training sufficient for the intervention & 13 & 0 \\
\hline Patients are comfortable with intervention & 12 & 1 \\
\hline \multicolumn{3}{|c|}{$\begin{array}{l}12 \text { nurses did not complete survey due to leave of absence } \\
{ }^{2} \text { Scale of } 1-10 \text { with } 10 \text { being the most comfortable and } 1 \text { being the least comfortable } \\
{ }^{3} \text { Scale of } 1-10 \text { with } 10 \text { being most likely and } 1 \text { being least likely }\end{array}$} \\
\hline
\end{tabular}

nurse practitioners applying and initiating radiographs in accordance with the OAR in triage. ${ }^{19}$ However, their study utilized nurse practitioners in a small Accident \& Emergency Department in which triage is covered by a single provider (NP or RN). In addition, it was not a RCT, but a case-control study, which may have systemic biases, such as patient volume in the ED at the time of triage that could confound results. When compared to the only other RCT related to the OAR and triage nurse initiation of radiographs, our results were much better. The Fan and Woolfrey study found a difference between control and intervention groups of only 6.7 minutes. ${ }^{20}$ This could be attributed to the fact that their study was done in an urgent care centre and not a tertiary care centre, where wait times can be much longer. The mean ED LOS in our study was 142.9 minutes and 114.7 minutes for the control group and OAR intervention group, respectively, compared to a mean ED LOS of 79.7 minutes and 73.0 minutes in control and intervention groups, respectively, in the Fan and Woolfrey study. It may be difficult to find a meaningful reduction in ED LOS when turnover of patients is more rapid, especially when there is no delay to physician assessment.
It is interesting to note that our results are consistent with a systematic review examining the role of triage nurse ordering. ${ }^{22}$ When examining injured patients with suspected fractures, triage nurse ordering intervention reduced ED LOS by 19 minutes in three RCTs, and by 18 minutes in five non-RCTs.

Although our finding of an improvement in throughput with OAR application at triage is consistent with our hypothesis and other studies mentioned, the improvement we found was less than anticipated and the potential reasons for this merit consideration. When the ED is not busy, patients get seen by EPs without delay; hence any potential time savings that triage nurse initiated radiography is anticipated to achieve would be lost, as patients are not spending prolonged periods of time in the waiting room. This may be partly the reason why the Fan and Woolfrey study failed to find a significant improvement in patients' ED LOS in their study. ${ }^{20}$ Unfortunately, we did not set a priori parameters to capture data to determine crowding in our ED at the time patients were enrolled in the study. Such a strategy would be preferable for future studies examining triage nurse ordering in the ED, as we suspect that our intervention of applying the OAR at triage would prove to be most useful during the busiest period in the ED, particularly when the longest wait element is time to provider. Despite the popularity of the OAR, the compliance of the rule by our group of EPs was poor, as $92 \%$ of the patients in our study received radiography, compared to $60 \%$ in the OAR implementation study by Stiell et al. ${ }^{2}$ This may be due to spectrum bias, as patients with mild injuries may now avoid coming to, or waiting in, a busy tertiary care centre due to extended wait times (a situation that has likely worsened since the publication of the implementation study), or it may be due to a lack of EPs' application of, or compliance with, the OAR. We suspect that after a patient's prolonged wait, EPs may feel compelled to justify the wait by ordering an $\mathrm{x}$-ray (and patients maybe more forceful in demanding this) rather than spending time explaining why an $x$-ray is unnecessary. If present, this behavior could undermine some or all of the effect of triage nurse screening and OAR application, especially with patients who were deemed OAR negative but who ended up with a prolonged stay due to EPs' initiation of radiographs. Finally, the setup and flow of the ED is a critical component in any transit improvement initiatives. Part of the limitation of our study was that 
patients needed to be registered after triage before a radiograph could be ordered. As a result, our triage nurses were not able to order radiographs in a timely fashion and often performed other triage-related duties before initiating the order. This may also have reduced the impact on ED LOS of applying the OAR at triage.

Generally, we found triage nurse initiated radiographs to be well accepted by patients. This was reflected by a small increase in patient satisfaction in the OAR group with regards to LOS ratings. As expected, there was no change in the quality of care ratings, as patients in both the control and the OAR group received standard EP assessment after triage. The intervention was also well received by 13 of our 15 triage nurses, as demonstrated by their willingness to continue applying the OAR after study completion, and their belief that it can reduce patient's ED LOS. Two triage nurses were on leave after completion of study and were not able to complete a nurse satisfaction survey. Seven of 13 surveyed triage nurses felt that implementation of the OAR increased their workload. This is not a surprising finding, as triage is known to be busy and even chaotic at times. If one adds a task to nurses at triage, one must consider what can be removed to avoid the unintended effect of other patients waiting longer to be triaged.

Our telephone survey indicated patients in the OAR group were more satisfied with their ED LOS. Both groups deemed a significant LOS reduction to be at least 30 minutes. This is in keeping with the literature, which suggests that shorter patient LOS in the ED correlates with improved patient satisfaction. ${ }^{17,18}$ Interestingly, we found quality of care ratings were similar in both groups, as was comfort level with triage nurse initiating $\mathrm{x}$-rays.

Our results strengthen the research suggesting that triage nurses can safely apply the OAR without any missed fractures. There was only one missed fracture in our study. However, that patient belonged to the control group, and therefore the missed fracture diagnosis was not due to improper triage nurse assessment and application of the OAR. Nevertheless, the agreement between EPs and triage nurses, as reflected by the kappa values of 0.46 for ankle OAR and 0.77 for foot OAR, was only moderate. This could be explained by either the EPs' bias of ordering radiographs in spite of the OAR results or triage nurses not appropriately applying the rules.

Interestingly, Fiesseler et al. found similar kappa values in their study, and concluded that nurses showed only a moderate ability to apply the OAR. ${ }^{15}$ Accurate triage nurse application of the OAR is important, as incorrect radiographs initiated at triage could paradoxically lead to increase in patients' ED throughput time, depending on circumstances at the ED in question. We believe that with sufficient training of triage nurses in application of the OAR, application errors can be minimized.

\section{LIMITATIONS}

Our study has limitations that should be considered. Physicians were blinded to negative OAR nurse assessments, but were not blinded to positive assessments requiring radiographs. Investigators were also not blinded to group allocation. Another limitation involves the patient satisfaction surveys. The large range of days prior to patient survey completion affects the accuracy of patients' recall. This large range was due to difficulties contacting patients with multiple telephone calls, limited to a maximum of five attempts, as well as lack of volunteer research students' availability. In addition, a validated patient satisfaction model, such as that established by Sun et al., ${ }^{23}$ was not used in this study. EP satisfaction was also not assessed. For patients who were lost to telephone follow-up, missed fractures could not be assessed. The computer database was checked for return visits to VGH and two other hospitals under the same health authority. However, it remains possible that some patients may have had return visits to another health authority.

Our study involved 15 motivated triage nurse volunteers, which may potentially result in a volunteer bias. As mentioned, the addition of the OAR at triage may increase workload for triage nurses, resulting in delay in triage for other ED patients. We did not specifically observe the added time needed for OAR assessment and ordering of radiographs at triage. Future studies could examine whether triage initiated radiographs contribute significantly to overall ED throughput time. Furthermore, currently at VGH, radiographs cannot be ordered until registration is completed. Ideally, future implementation of the OAR would require the ability to streamline our image ordering process. Other institutions may already have joint triage and registration, which would obviate this difficulty.

Finally, it should be noted that there was an imbalance in the number of CTAS $3 \quad(36.6 \%$ vs $21.3 \%)$ and $4(63.4 \%$ vs $78.4 \%)$ between the OAR and control 
group. However, we feel it is unlikely this affected our results, as in the ED ambulatory care area of our institution, no distinction is made in the processing of CTAS 3 and CTAS 4 patients. Patients are by and large seen by time of arrival, unless classified as CTAS 2. It is more difficult to assess any potential impact of the imbalance in patient arrival times between the OAR and control group. We suspect that what is most significant is the state of the department at the time of patient arrival. It remains possible that the intervention studied may have a different impact based on where the maximal bottleneck is, and more specifically whether it is at triage or time to EP.

By having a small group of triage OAR nurses participating in our study, we were not able to enroll all eligible candidates presenting to the ED during the study period. Fifteen triage nurse participants as well as our RCT design did, however, provide adequate consistency and ensured that the rules were applied during days, nights, weekends, and weekdays, as well as during high and low ED volume days. Finally, this study was conducted in a busy tertiary care centre, in a province where ordering of $\mathrm{x}$-rays is part of nurses' scope of practice, and thus our findings may not be generalizable to all settings.

\section{CONCLUSION}

Triage nurse initiated radiograph using the OAR is accepted by both nurses and patients, and led to a statistically significant decrease of 20 minutes median and 28 minutes mean ED LOS at a tertiary care centre. As the impact from this intervention is undoubtedly site-dependent, other EDs should consider whether implementation of this intervention at triage would result in a significant reduction in patients' ED LOS, while at the same time assessing for potential delays in triage caused by the OAR application.

\section{Competing Interests: None to declare.}

Acknowledgment: We thank our 15 volunteer OAR nurses and Ms. Janice Rotinsky, our Nurse Educator, without whom this study would not have been possible.

\section{REFERENCES}

1. Stiell IG, Greenburg GH, McKnight RD, et al. Decision rules for the use of radiography in acute ankle injuries: refinement and prospective validation. $7 A M A$ 1993;269(9):1127-32.

2. Stiell IG, McKnight RD, Greenburg GH, et al. Implementation of the Ottawa Ankle Rules. FAMA 1994;271(11):827-32.
3. Stiell I, Wells G, Laupacis A, et al. A multicentre trial to introduce clinical decision rules for the use of radiography in acute ankle injuries. BM7 1995;311(7005):594-7.

4. Anis AH, Stiell IG, Steward DF, et al. Cost-effectiveness analysis of the Ottawa Ankle Rules. Ann Emerg Med 1995; 26(4):422-8.

5. Bachmann LM, Kolb E, Koller MT, et al. Accuracy of Ottawa ankle rules to exclude fractures of the ankle and midfoot: systematic review. BM7 2003;326(7388):417.

6. Broomhead A, Stuart P. Validation of the Ottawa Ankle Rules in Australia. Emerg Med (Fremantle) 2003;15(2):126-32.

7. Yuen MC, Sim SW, Lam HS, et al. Validation of the Ottawa ankle rules in a Hong Kong ED. Am 7 Emerg Med 2001;19(5):429-32.

8. Auleley GR, Kerboull L, Durieux P, et al. Validation of the Ottawa ankle rules in France: a study in the surgical emergency department of a teaching hospital. Ann Emerg Med 1998;32(1):14-8.

9. Tay SY, Thoo FL, Sitoh YY, et al. The Ottawa Ankle Rules in Asia: validating a clinical decision rule for requesting $\mathrm{X}$-rays in twisting ankle and foot injuries. 7 Emerg Med 1999;17(6):945-7.

10. Yazdani S, Jahandideh H, Ghofrani H. Validation of the Ottawa Ankle Rules in Iran: a prospective survey. $B M C$ Emerg Med 2006;6:3.

11. Karpas A, Hennes H, Walsh-Kelly C. Utilization of the Ottawa ankle rules by nurses in a pediatric emergency department. Acad Emerg Med 2002;9(2):130-3.

12. Hopkins M. A comparative analysis of ENP's and SHO's in the application of the Ottawa ankle rules. Inter Emerg Nurs 2010;18(4):188-95.

13. Salt $\mathrm{P}$, Clancy M. Implementation of the Ottawa ankle rule by nurses working in an accident and emergency department. 7 Accid Emerg Med 1997;14(6):363-5.

14. Mann CJ, Grant I, Guly H, et al. Use of Ottawa ankle rules by nurse practitioners. 7 Accid Emerg Med 1998; 15(5):315-6.

15. Fiesseler F, Szucs P, Kec R, et al. Can nurses appropriately interpret the Ottawa ankle rule. Am 7 Emerg Med 2004; 22(3):145-8.

16. Derksen RJ, Bakker FC, Geervliet PC, et al. Diagnostic accuracy and reproducibility in the interpretation of Ottawa ankle and foot rules by specialized emergency nurses. Am $\mathcal{Z}$ Emerg Med 2005;23(6):725-9.

17. Preyde M, Crawford K, Mullins L. Patient satisfaction and wait times at Guelph General Hospital Emergency Department before and after implementation of a process improvement project. CFEM 2012;14(3):157-68.

18. Welch SJ. Twenty years of patient satisfaction research applied to the emergency department: a qualitative review. Am 7 Med Qual 2010;25(1):64-72.

19. Allerston J, Justham D. A case-control study of the transit times through an accident and emergency department of ankle injured patients assessed using the Ottawa ankle rule. Accid Emerg Nurs 2000;8(3):148-54.

20. Fan J, Woolfrey K. The effect of the triage-applied Ottawa ankle rule on the length of stay in a Canadian urgent care department: a randomized controlled trial. Acad Emerg Med 2006;13(2):153-7. 
21. Parris W, McCarthy S, Kelly AM, et al. Do triage nurseinitiated X-ray for limb injuries reduce patient transit time? Accid Emerg Nurs 1997;5(1):14-5.

22. Rowe B, Villa-Roel C, Guo X, et al. The role of triage nurse ordering on mitigating overcrowding in emergency department: a systematic review. Acad Emerg Med 2011; 18(12):1349-57.

23. Sun BC, Adams JG, Burstin HR. Validating a model of patient satisfaction with emergency care. Ann Emerg Med 2001;38(5):527-32. 